\title{
Properties of biodegradable films based on thermoplastic starch and poly(butylene succinate) with plant oil additives
}

\author{
$\underline{\text { Piotr Tynski }}^{1}$, Justyna Ostrowska ${ }^{2}$, Magdalena Paluch $^{3}$, Waldemar Sadurski ${ }^{4}$
}

1. Organic Technology Department, New Chemical Syntheses Institute, POLAND, 24-110 Puławy, Al.Tysiąclecia Państwa Polskiego 13A, E-mail: piotr.tynski@ins.pulawy.pl

2. Organic Technology Department, New Chemical Syntheses Institute, POLAND, 24-110 Puławy, Al.Tysiąclecia Państwa Polskiego 13A, E-mail: justyna.ostrowska@ins.pulawy.pl

3. Organic Technology Department, New Chemical Syntheses Institute, POLAND, 24-110 Puławy, Al.Tysiąclecia Państwa Polskiego 13A, E-mail: magdalena.paluch@ins.pulawy.pl

4. Organic Technology Department, New Chemical Syntheses Institute, POLAND, 24-110 Puławy, Al.Tysiąclecia Państwa Polskiego 13A, E-mail: waldemar.sadurski@ins.pulawy.pl

The paper presents study of the plant oils effect on the properties of starch/polyester blends. In the first stage of the study thermoplastic starch (TPS) with different amount of oils were prepared. I the second stage TPS materials were blended by extrusion process with commercially available biodegradable polyesters - poly(butylene succinate).

Keywords - thermoplastic starch, poly(butylene succinate), blends, biodegradable films.

\section{Introduction}

The growing interest in using eco friendly products has stimulated research and development of new materials such as biodegradable polymers. Furthermore, due to various restrictions regulated by the law and a very high consumption of plastics, fluctuating cost of petroleum-based materials based on oil prices and environment pollution, biodegradable polymers have been developed and have partially or completely replaced conventional plastics in some applications.

Starch is an abundant and naturally occuring polymer that is increasingly used in the production of packaging. Starch has been considered as an attractive biopolymer because of its low cost, low density and biodegradability. It can be used in its native form as a filler with another polymer or it can be processed into a thermoplastic material and mixed with other polymers to forms polymer blends [1,2].

Native starch is found in granular form and it is composed of two major polysaccharides, amylose and amylopectin (Fig. 1). Linear amylose is soluble in water and has a helical structure. Amylopectin molecules are short, branched and easy to crystallize.

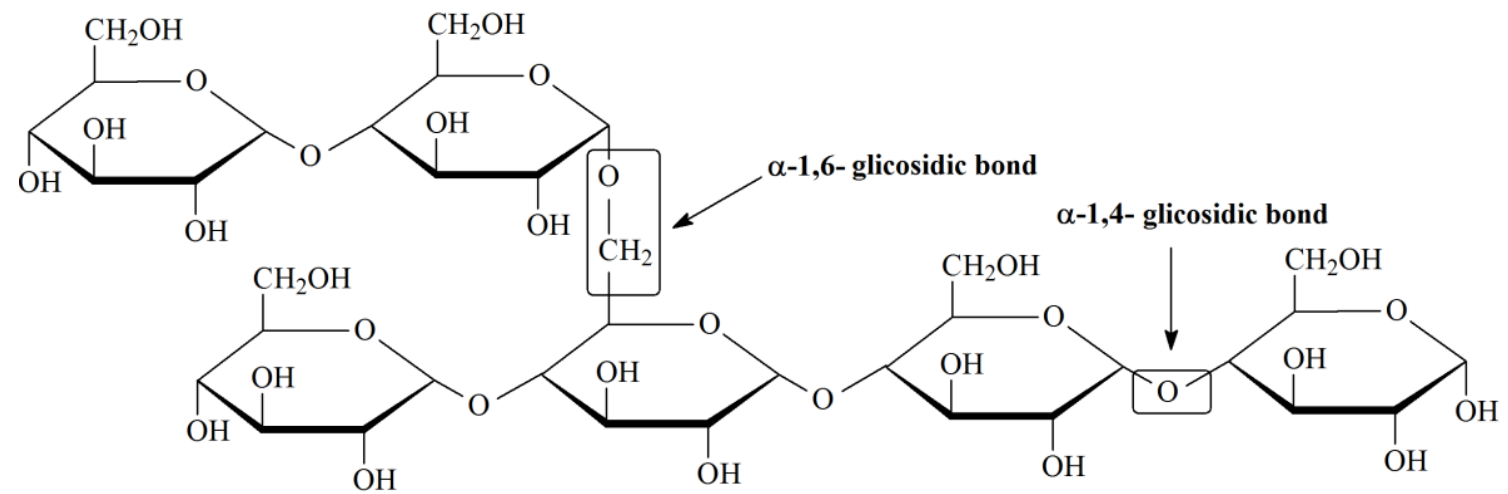

Figure 1. Chemical structure of starch. 
Starch granules exhibit hydrophilic properties and strong itermolecular association via hydrogen bonding due to the hydroxyl groups. The melting point of native starch is higher than the thermal decomposition temperature, hence starch cannot be thermally processed in native form. To resolve this problem, non-volatile plasticizers such as polyol (glycerol, ethylene glycol etc.) are commonly used. For this purpose, the plasticizers is added to starch, and then the material is processed in the extruder at higher temperatures and shear forces. In this way, the crystal structure is destroyed and replaced by starch hydrophilic thermoplastic material with an amorphous structure which can be processed like other thermoplastics by extrusion process. A new material obtained from native starch with plasticizers is called thermoplastic starch (TPS) [3].

Industrial applications of starch based materials are limited by the susceptibility of their final properties to certain environment changes. They present poor mechanical properties and water vapour barrier capacity, compared to synthetic polymers. Thus, blend formation by adding compatible materials represents a promising alternative to improve the final properties reducing the sensitivity to environment parameters like humidity, temperature or radiation exposure. Within this context, the use of a hydrophobic biodegradable polymer such as polylactide (PLA), poly(butylene succinate) (PBS) or poly( $\varepsilon$-caprolactone) (PCL) for starch-based blends would offer the possibility of enhancing mechanical behavior and reducing water vapour permeability $[4,5]$.

Poly(butylene succinate) PBS is one of the promising hydrophobic aliphatic polyester (Fig. 2) with many interesting properties including biodegrability, melt processability and thermal and chemical resistance. PBS can be processed in the field of textiles into melt blow, multifilament, flat and split yarn and also in the field plastics into injection molded products. The characteristics of PBS are simililar to those of polyethylene. It has good elongation at break and low melting point with ductile property that can be easily applicable to conventional processing techniques such as extrusion and injection molding.

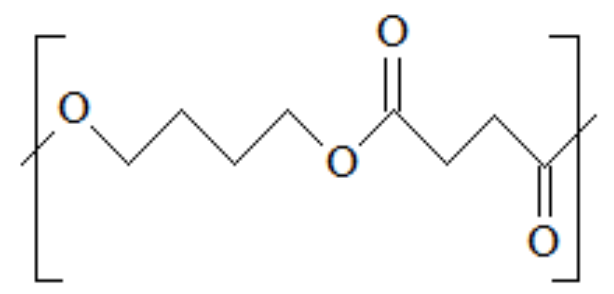

Figure 2. Chemical structure of poly(butylene succinate).

One of limitaions regarding the use of PBS in various commercial application is its high cost. Blending with low-price resins like thermoplastic starch is an alternative approach to resolve those problems. Hovewer, it was confirmed that TPS/PBS blends were immiscible and have the poor compatibility. Theer are very few reports about TPS/PBS blends and their compatibilization. One of the possibilites is to obtain the reactive PBS. It was found that the mechanical properties of blends improved and reduced water absorption with the increase of the reactive PBS content in blend [6]. Another options to improve properties of hydrophilichydrophobic blend is to incorporate the additives that will modify the interfacial properties of the polymers. The compatibilizing additives can be amphiphilic compounds which in their structure 
have hydrophilic and hydrophobic groups. The most common amphiphilic compounds are fatty acids, lipids, triglycerides, surfactants or proteins [7,8].

The paper presents the study on the influence of the addition of amphiphilic compounds contained in rapeseed oil and blackcurrant seed extract on selected properties of blends and films based on thermoplastic starch (TPS) and poly(butylene succinate) (PBS).

\section{Experimental part}

In the first stage of the research, a thermoplastic starch in the granulate form containing various concentrations of rapeseed oil or blackcurrant extract using twin screw co-rotating extruder was obtained. Then, the TPS granulate was blended in extrusion process with commercially available biodegradable polyester - poly(butylene succinate) (PBS). The blend containing $50 \mathrm{wt} \%$ of TPS was prepared. In order to determine the properties of obtained polymer granules, water content in granulate, FTIR spectroscopy and melt flow index (MFR) were analysed.

In the second stage of the research, films on a large-scale scale from the prepared materials by extrusion blow molding were obtained. The extrusion processes for individual mixtures were optimized and tests to determine the mechanical properties (tensile strength and elongation at break) of the obtained films were carried out.

\section{Results and disscusion}

\section{Water content and melt flow index (MFR)}

Determination of water content in biogranulates was carried out by Karl Fischer volumetric titration method at $120^{\circ} \mathrm{C}$. The melt mass flow rate (MFR) was measured by using a plastometer CEAST MF20 apparatus at $190^{\circ} \mathrm{C}$ with $2,16 \mathrm{~kg}$ nominal load adapter to the standard ISO 1133.

Table 1. Results of the water content and mass melt flow index (MFR) of the TPS/PBS

\begin{tabular}{|c|c|c|}
\hline & \multicolumn{2}{|c|}{$50 \%$ TPS + 50\% PBS } \\
\hline & $\begin{array}{r}\text { water content } \\
{[\%]}\end{array}$ & $\begin{array}{c}\text { MFR } \\
{[\mathrm{g} / 10 \mathrm{~min}]}\end{array}$ \\
\hline without additive & 1,6 & 1,9 \\
\hline $0,5 \% \mathrm{BC}$ & 1,9 & 1,7 \\
\hline $1,0 \% \mathrm{BC}$ & 3,6 & 2,4 \\
\hline $1,5 \% \mathrm{BC}$ & 3,5 & 3,3 \\
\hline $0,5 \% \mathrm{RO}$ & 1,5 & 1,8 \\
\hline $1,0 \% \mathrm{RO}$ & 2,4 & 2,7 \\
\hline $1,5 \% \mathrm{RO}$ & 2,5 & 2,3 \\
\hline
\end{tabular}

The water content in the obtained blends is an important parameter in their further processing. During the production of the film from individual mixtures by blow molding, the effect of water content in the granulate on the structure of the film was observed. On the one hand, the minimum amount of water is necessary for the easier processing, and on the other hand, if the amount of water in the granulate from which the film is extruded is too small, inclusions of starch granules occur. Obtaining the optimum amount of water in the granulate of the blend determines the production of the film without disadvantageous inclusions and with a satisfactory efficiency. 
The simplest measurement of the rheological properties of thermoplastic polymers is the determination of the mass or volume melt index of plastics using a plastometer. Knowing the value of the melt flow index of a material, it is possible to select the processing methods.

The following table summarizes the results of the water content and mass melt flow index (MFR) for individual granulates.

The water content in the obtained granules determines the production of a film with good structural properties. The value of the melt flow index (MFR) obtained for individual TPS/PBS granulates enables processing by extrusion blow molding and films production.

\section{FTIR spectroscopy}

FTIR spectra were measured using Nicolet iS10 Spectrum Scanner (Thermo Scientific, USA) over the wavenumber ranging from 600 to $4000 \mathrm{~cm}^{-1}$ using ATR-FTIR mode system. Figure 3 shows overlaid FTIR spectra of TPS/PBS and blend with 1.5\% oil additive.

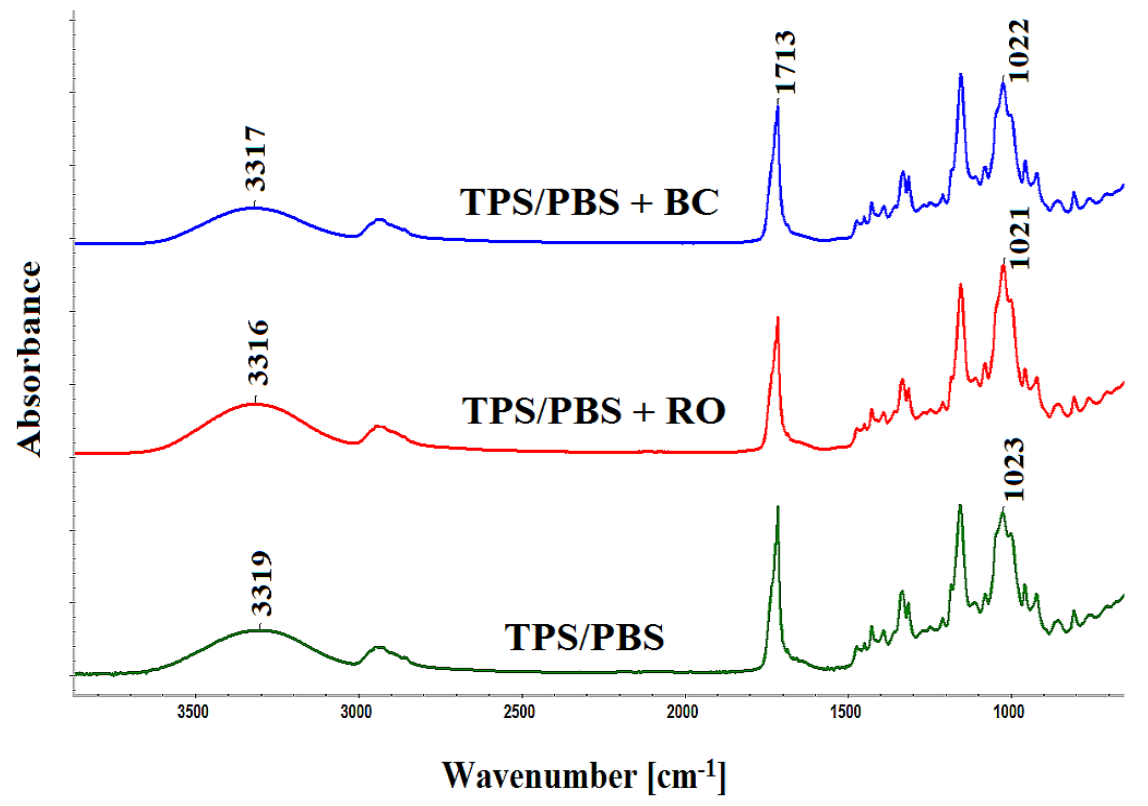

Figure 3. FTIR specta of TPS/PBS blends.

As a result of the addition of $1.5 \%(\mathrm{BC})$ or (RO) oils to the TPS/PBS blend, the band at $1020 \mathrm{~cm}^{-1}$ derived from the vibrations of the $\mathrm{C}-\mathrm{O}$ groups in the glucose ring occurring in the starch structure increases its intensity. This is the effect of interactions between the hydrophobic groups in the oil structure and the chains of the hydrophobic polyester. The $\mathrm{C}-\mathrm{O}$ groups vibration band in the spectrum of oil-containing blends is shifted from 1023 to $1022 \mathrm{~cm}^{-1}$ (BC) and $1021 \mathrm{~cm}^{-1}$ (RO), confirming the improvement in the miscibility between the hydrophobic PBS polyester and the hydrophilic thermoplastic starch. An increase in the intensity of the hydrogen bond band at about $3300 \mathrm{~cm}^{-1}$ in the TPS/PBS blends containing the oil additive (BC) or (RO) and its shift towards the lower wavenumbers was also observed. This is due to the fact that the carbonyl group $\mathrm{C}=\mathrm{O}$ present in the oil compounds structure forms additional hydrogen bonds with the hydroxyl groups of the starch. 


\section{Mechanical properties}

The mechanical properties of the materials were determined using a universal testing machine Instron 3340 according to the ISO 527 standard. Figure 4 shows the dependence of the tensile strength and elongation at break for the TPS/PBS films modified by different type of oils. The addition of rapeseed oil (RO) and blackcurrant extract (BC) results in a significant improvement in the tensile strength of films compared to TPS/PBS blends without oils additives. Moreover, elongation at break parameter increases by approx. 300\%. This indicates that films with amphiphilic compounds are more flexible.The optimal value of the additives are $0,5 \%$, because for larger quantities of oil additives, the mechanical parameters are reduced in relation to the properties of the film with the addition of $0,5 \%$.

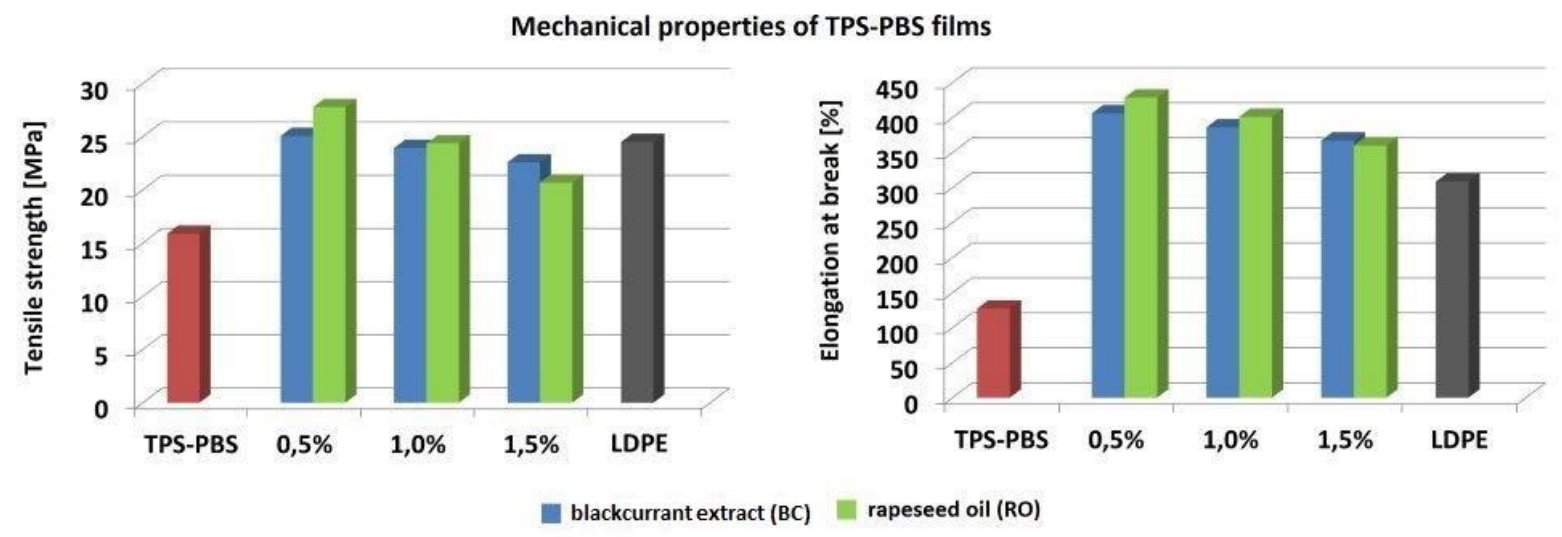

Figure 4. Tensile strength and elongation at break of TPS - PBS films.

\section{Conclusions}

Film blowing of TPS - polyester blends containing $50 \mathrm{wt} \%$ thermoplastic starch after optimization runs stably and smoothly on standard blown film extrusion lines. TPS - PBS films with added oils exhibited significantly improved mechanical properties. The most significant improvement of films properties was observed in the case of oil additive in an amount of $0,5 \mathrm{wt}$ $\%$. The improved mechanical properties of TPS - PBS films with oil additive showed better miscibility between the polymers.

\section{References}

[1] V. Siracusa, P. Rocculi, S. Romani, M. Dalla Rosa, "Biodegradable polymers for food packaging: a review", Trends in Food Science \& Technology, vol. 19, no. 12, December, pp. 634-643, 2008.

[2] L.P.B.M. Janssen, L. Mościcki (eds.), Thermoplastic Starch: A Green Material for Various Industries, WILEY-VCH Verlag GmbH \& Co. KGaA, Weinheim, 2009

[3] M. Kaseem, K. Hamad, F. Deri, "Themoplastic starch blends: A review of recent works", Polymer Science, vol. 54, pp. 165-176, 2012.

[4] L. Averous, L. Moro, P. Dole, C. Fringant, "Properties and thermoplastic starch blends: Starch-polycaprolactone" Polymer, vol. 41, no. 11, pp. 4157-4167, 2000.

[5] M. D. Ninago, O. V. Lopez, M. M. S. Lencina, M. A. Garcia, N. A. Andreucetti, A. E. Ciolino, M. A. Villar "Enhancement of thermoplastic starch final properties by blending with poly(E-caprolactone)" Carbohydrate Polymers, vol. 134, pp. 205-2012, 2015. 
[6] J. Zeng, L. Jiao, Y. Li, M. Srinivasan, T. Li, Y. Wang, "Bio-based blends of starch and poly(butylene succinate) with improved miscibility, mechanical properties, and reduced water absorption", Carbohydrate Polymers, vol. 83, pp. 762-768, 2011.

[7] R.P.H. Brandelero, M.V. Grossmann, F. Yamashita, „Films of starch and poly(butylene adipate co-terephthalate) added of soybean oil (SO) and Tween 80", Carbonhydrate Polymers, vol. 90, no. 4, November, pp. 1452-1460, 2012.

[8] R. Ortega-Toro, A. Jimenez, P. Talens, A. Chiralt, „Effect of the incorporation of surfactants on the physical properties of corn starch films", Food Hydrocolloids, vol. 38, July, pp.66-75, 2014. 